the global
impairment
was more
severe in
PD than in
essential
tremor

MOVEMENT DISORDERS

\title{
Comparison of cognitive impairment in Parkinson disease and essential tremor
}

\author{
A gradient of global cognitive \\ impairment exists between healthy \\ controls, patients with essential \\ tremor and patients with Parkinson \\ disease (PD), according to a new \\ study. Performance in multiple \\ domains revealed overlapping but \\ distinct patterns of impairment in \\ the two disorders. \\ Cognitive impairment often \\ accompanies PD and essential \\ tremor, and the impairments are \\ similar - they usually affect atten- \\ tion, verbal fluency and memory. \\ Direct comparisons between the \\ impairments in the two conditions \\ have so far been limited to just three \\ small studies in which patients with \\ dementia were not excluded. In their \\ new study, Álvaro Sánchez-Ferro and \\ colleagues aimed to address the limi- \\ tations of the previous populations. \\ "This project was part of a large \\ population-based study called \\ Neurologic Disorders in Central \\ Spain (NEDICES), and we investi- \\ gated cognitive deficits in people \\ affected by essential tremor and PD \\ in the absence of dementia," explains
}

Sánchez-Ferro. "We compared the two diseases to gain further insight into the impairment that exists at the early stages of these disorders."

The study included 180 patients with essential tremor, 46 with PD, and 2,212 healthy controls. Participants had taken tests of global cognitive performance, cognitive processing speed, verbal fluency, memory and premorbid intelligence, and the researchers used nonparametric tests to compare the scores.

The analysis revealed that patients with essential tremor and patients with PD were cognitively impaired relative to healthy controls, and that the global impairment was more severe in PD than in essential tremor, indicating a gradient of impairment. A head-to-head comparison of performance in the two conditions showed that this trend held true in tests of memory and verbal fluency, although the differences were not significant. By contrast, patients with essential tremor consistently performed worse than patients with $\mathrm{PD}$ on tests of cognitive processing speed, indicating distinct patterns of impairment in the two conditions.

"To adjust for confounding effects, we performed a series of subanalyses that gave us enough confidence to attribute our findings to the diseases and not to factors such as age or literacy," states Sánchez-Ferro.

The results require confirmation in further studies, but Sánchez-Ferro says that, if replicated, the findings will help us to understand the pathophysiology of essential tremor and PD and will highlight the fact that the symptoms of essential tremor are not restricted to motor function.

"The findings could also pave the way to develop novel methods to differentiate these two disorders, which can have substantial overlap," concludes Sánchez-Ferro.

\section{Ian Fyfe}

ORIGINAL ARTICLE Sánchez-Ferro, Á. et al. Cognition in non-demented Parkinson's disease versus essential tremor: a population-based study. Acta Neurol. Scand. http://dx.doi.org/10.1111/ ane.12752 (2017)

FURTHER READING Aarsland, D. et al. Cognitive decline in Parkinson disease. Nat. Rev. Neurol. http://dx.doi.org/10.1038/nrneurol.2017.27 (2017) 